\title{
EFFECT OF DENSITY CORRELATIONS ON THE COHERENCY OF RELATIVISTIC BUNCH RADIATION
}

\author{
October 25, 2018

\begin{abstract}
The coherent radiation of the electron beam with account of particle position correlations because of strong Coulomb interaction is considered. For the first time it is shown that the coherency of the radiation of the bunch with account of correlations depend on the mean inter particle distance (mean density of the bunch). The conditions of coherency are found. The influence of deviations from mean distance on the radiation coherency is considered.
\end{abstract}

R. V. Tumanian *, L. A. Gevorgian, Yerevan P

Report at NATO Workshop Nor-Hamberd, Yerevan, Armenia, 25-29 June, Ed. by H. Wiede

\section{Introduction}

In recent years very high density or small size bunches become to possible to obtain I1, 2. It is clear that in such dense bunches the self space charge forces of the bunch particles (electrons) play significant influence on the bunch parameters and particles motion. However, in this paper we investigate the useful effects of the space charge instead of deleterious effects of that. In the last two decades very important experimental [9, 10] and theoretical [?] results have been achieved in investigations of the ordered or crystalline beams. The possibility of such new and interesting state of matter is become real in the storage rings of charged particles and as we show below in linear accelerators with high density beams too. The case of real storage ring lattice was considered in [15], where it was shown that strong cooling is necessary for attaining crystalline beams in the storage rings. In difference from storage rings in the 18 it was shown that energy spread,sizes and particles motion of the linac's beams at high energies are not sensitive to longitudinal space charge effects for existing beam currents.

*raphael@star.yerphi.am 
The crystalline or ordered beam have of course many interesting and important properties and applications. A few properties and one very important application is considered in this paper. It is clear that particles of dense bunches may be arranged in the certain orders. In these ordered bunches particles form transverse planes, which are same spacing in the longitudinal direction. The conditions and properties of such ordering are considered in the next section of this paper. Such bunches are radiate coherently at the wavelengths integer times smaller than interplane distances, as shown in section 3 of this report. The importance of tunable and powerful sources of the coherent radiation of XUV wavelengths has stipulated several projects of FEL in recent years [1, 2]. Those FEL's operate in the Self Amplified Spontaneous Emission (SASE) mode, i.e. starting from noise in the initial distribution of the electron beam longitudinal density. This evolution determines the undulator length needed to reach saturation practically to be very long (about hundred meters). The necessary power of laser radiation is possible to obtain by using ordered bunches. This radiation calls superradiant regime of FEL radiation [?] or coherent spontaneous emission (CSE). As shown in above references it is possible in two cases. First, for short bunches and second for modulated bunches. The superradiant regime due to self bunching of the beam in the FEL is considered by Bonifacio [6, 7]. In difference from above references where is considered the coherency of long wavelength radiation (the wavelength much more than mean distance between bunch electrons), in this report is considered the coherent radiation of the relativistic bunch when the radiation wavelength is about or less than distance between particles. In this case it is important the discreteness of beam and correlations between beam particles positions, because of strong Coulomb interaction of the beam particles.

\section{Correlations and Ordering of the Relativistic Bunches}

\subsection{Introduction}

The requirement of bunch uniformity assumes, that electrons of the bunch with density $\mathrm{n}$ are placed on the same mean distance $a=n^{-1 / 3}$ from each other. Such a placement is possible only when each three of particles compose equilateral triangle as a result of strong correlation between particles positions. This is connected with approximately hexagonal structure of the disordered medium [8] as the most probable. Each particle in a such medium have 14 nearest neighbors, but numerical calculations show that the mean number of nearest neighbors is about 15 with mean deviation about 10 percents. For medium with the long distance interparticle interaction such as beam or bunch the vertices of triangles are equilibrium points of the particle positions. Particles which are not placed in their equilibrium points oscillate as shown below around their equilibrium 
positions with amplitudes equal to deviations from equilibrium points. It is well known that properties of any medium depend on Madelung energy - dimensionless parameter $\Gamma$, which is the ratio of the depth of the interparticle potential well and medium temperature. For neutral and one component plasma (OCP) due to the Debay screening interparticle potential is about bare two particle Coulomb potential $e^{2} / a$ 14. The numerical Molecular Dynamics (MD) calculations show that for $\Gamma \geq 172$ OCP is crystallize with body centered cube (bcc) lattice 13]. Detailed MD calculations 11] for finite full charged Coulomb systems with number of particles about thousand was show that in this case crystallization takes place at low values of $\Gamma$, but this result has not been explained theoretically yet. We show in this report that for Coulomb systems of high energy charges the potential well of each particle is for about $N_{t}$ (number of particles in the one transverse plane of the bunch) times deeper than that of the bare two particle Coulomb potential. In contrast to crystalline beams in the storage rings, where strong cooling is necessary for attaining crystalline state [15], as shown in the [18] a such beam optics may be designed that energy spread, particle dynamics and sizes of the bunch in the Final Focus of the linac are sensitive to longitudinal space charge effects only for bunches with $N \succeq 10^{11}$. Calculations show that the beam of the SLAC in the Final Focus have $\Gamma$ for about a few hundreds that means that bunch may be crystallized. Note, that in this case resonances with betatron oscillations are not important because of longitudinal ordering of the bunch and disordering in the transverse directions. Classical consideration is valid, when $\hbar \Omega \ll T=m c^{2} \delta^{2}$, with $\delta=\frac{\Delta E}{E}$ relative

energy spread.This condition is equal to $\varepsilon=\frac{\lambda_{c} \sqrt{\Gamma}}{a \delta \gamma^{3 / 2}} \ll 1$, which is satisfied for $\gamma \sim 10^{4}$. In this case bunch become longitudinal ordered as considered in the section 2.3. When the condition $\varepsilon \leq 1$ satisfied the quantum effects should be taken in the account and results of the [16, 17] can be used for bunch electrons correlation function. This case is considered in the section 2.2.

\subsection{Correlations of the Strong Interacting Charges En- sembles}

The statistical properties of $\mathrm{N}$ particle ensemble are described by N-particle distribution function $f_{N}$, which is depend generally on positions and momentums of all $\mathrm{N}$ particles of the ensemble. As seen below for calculating of the radiation coherency the two particle distribution function

$$
f_{2}=\int d^{N-2} x d^{N-2} p f_{N}
$$

, or the correlation function is necessary

$$
f_{2}=g_{2}\left(x_{1}, x_{2}\right)+f_{1}\left(x_{1}\right) f_{1}\left(x_{2}\right)
$$

. Charges in the bunches are strong correlated because of strong Coulomb interaction. As considered above, quantum effects should be taken into account, 
when $\varepsilon$ do not much less than unity. In this case we can use the density correlation functions obtained in the 16, 17 for strong correlated electrons

$$
\langle\varrho(x) \varrho(0)\rangle \approx \cos \left(2 \pi \frac{x}{a}\right) \exp [-b \sqrt{\ln (x)}]
$$

where $b=\frac{\varepsilon}{\Gamma}, \mathrm{m}$ is effective mass, in the units $\hbar=1$.It is clear that $b \ll 1$ and one can obtain from this correlation function the approximate two particle distribution function

$$
g_{2}\left(x_{1}, x_{2}\right)=\left(1+\cos 2 \pi \frac{x}{a}\right) / l
$$

where $l$ is the bunch length. This is true in the liquid or partially ordered state of the bunch. Such state is possible to obtain at energies of the electrons for about tens Mev, if the bunch have energy spread for about percents and number of particles about $10^{10}$, which is about ten times greater than that in SUNSHINE [19]

\subsection{Ordering of the Charges in the Relativistic Bunch}

After expanding of the full force between particles with charge e and fixed distance $\mathrm{R}$ moving along longitudinal $\mathrm{z}$ direction with velocity $v=\beta c$ and angle $\theta$ between $\mathrm{R}$ and $\mathrm{z}$ directions,

$$
\begin{gathered}
F_{z}=\frac{e^{2}}{R^{2}} \frac{\left(1-\beta^{2}\right) \cos \theta}{\left(1-\beta^{2}(\sin \theta)^{2}\right)^{3} / 2} \\
F_{\perp}=\frac{e^{2}}{R^{2}} \frac{\left(1-\beta^{2}\right) \sin \theta}{\left(1-\beta^{2} \sin ^{2} \theta\right)^{3 / 2}}
\end{gathered}
$$

around equilibrium points of the bcc lattice,one can find that potential wells in the longitudinal and transverse directions are

$$
\begin{aligned}
U_{0 \|} & =U_{0} \frac{N_{t}}{4 \gamma^{2}} S_{l} & , \quad S_{l} & =\sum \frac{1}{n^{3}} \\
U_{\perp} & =U_{0} \frac{a_{l}}{a_{t}} \frac{\gamma}{4} S_{t} & , \quad S_{t} & =\sum \frac{n_{1}}{\left(n_{1}^{2}+n_{2}^{2}\right)^{2}}
\end{aligned}
$$

where $U_{0}=e^{2} / a$ is the bare Coulomb potential, $N_{t}$ is the number of particles on each transverse plane. The sum in the $S_{l}$ is carrying out over number of transverse planes ( $n_{3}$ denotes the plane number), and the sum in the $S_{t}$ over $n_{1}, n_{2}$ which numerate particles in $\mathrm{x}, \mathrm{y}$ directions respectively on the each plane.Here is assumed that self space charge forces of the bunch are compensated by external restoring forces 11, 12]. In this case particles oscillate in the potential

$$
U_{z}=U_{0 \|} \frac{z^{2}}{a^{2}}
$$

Such deepening of the potential well in the bunches in comparison with known potential well in the OCP means that crystallization or ordering of bunch is 
possible at comparable higher temperatures than of OCP. This we proof by consideration of the canonical partition function of the Gibb's canonical ensemble with hamiltonian $H_{N}$ and inverse temperature in energy units $\beta=1 / k T$ :

$$
Q_{N}(\Omega, \beta)=\frac{1}{h^{3 N} N !} \int d r^{N} d p^{N} \exp \left(-\beta H_{N}\left(r^{N}, p^{N} ; \Omega\right)\right)
$$

for a system of $\mathrm{N}$ charges enclosed in a volume $\Omega$; here h is Planck's constant. In the approach used by us hamiltonian $H_{N}$ of $\mathrm{N}$ relativistic charges is represented in the usual classical form as a sum of kynetic $E_{N}$ and potential $U_{N}$ energies $H_{N}=E_{N}+U_{N}$. Total potential energy $U_{N}$ equal to the sum of one particle potentials 7. In this approach the usual Helmholtz free energy $F_{N}(\Omega, \beta)$ as thermodynamic potential

$$
Q_{N}(\Omega, \beta)=\exp \left(-\beta F_{N}(\Omega, \beta)\right)
$$

and dimensionless configuration integral

$$
Z_{N}(\Omega, \beta)=\int \frac{d r^{N}}{\Omega^{N}} \exp \left(-\beta U_{N}\left(r^{N}, \Omega,\right)\right)
$$

It is easily seen from this equation that $Z_{N}$ depends on $\beta$ and on interactions only through the dimensionless combination $U_{0 \|} \beta$, which is the usual OCP $\Gamma$ multiplied by $N_{t} / \gamma^{2}$. So, the $\Gamma$ for dense enough ensemble of relativistic charges (bunch), can be greater than $\Gamma$ for OCP. Particle of the bunch in this potential well is oscillate with frequency

$$
\Omega^{2}=\frac{U}{2 m a^{2}}
$$

where $\mathrm{a}$ is the mean interparticle distance in correspondence direction, $\mathrm{m}$ is the effective mass of bunch particle equal to $m \gamma^{3}$ for linacs. This formula is obtained from equation of particle motion in the longitudinal potential 7

$$
m \ddot{z}+\frac{U_{0 \|}}{a^{2}} z=0
$$

The case of real storage ring lattice was considered in 15. If the crystallization time which equal few oscillation periods less than period of betatron oscillation of the accelerator the bunch becomes ordered. In the harmonic approach the potential in the longitudinal direction is equal

$$
U_{\|}=U_{0 \|} \frac{z^{2}}{2 a^{2}}
$$

This formula show that the decreasing of the $a$ means increasing of the potential well. Such decreasing of the interparticle distance take place in the linacs due to adiabatic decreasing of the bunch sizes or bunch compression. Notice, that 
since magnetic compression do not change full energy, the bunch temperature (mean kinetic energy in the rest frame) must be decreases due to ensemble full potential energy (which is positive for the ensemble of one sign charges) increasing because of compression. More detailed analyze show that during acceleration in the linac $\Gamma$ is changes in correspondence with dependence

$$
\Gamma \sim \gamma^{1 / 2} l^{1 / 2}
$$

Such result is obtained from the assumption of adiabatic changing of the energy $\gamma$ and bunch longitudinal size l. This is possible in the case large $\Omega$, which may be obtained after sufficient compression of the bunch.

\section{Coherence Radiation of the Bunch}

\subsection{Introduction}

Radiation of the ensemble of $\mathrm{N}$ electrons moving along identical trajectories but with arbitrary spatial placement may be written in the form [3]

$$
I=I_{0} N(1+(N-1) F)
$$

where $I_{0}$ is the intensity of single electron radiation, $\mathrm{N}$ is the number of the electrons in the bunch, $\mathrm{F}$ is the factor of coherency of the bunch and may be written in the follow form [3

$$
F=\frac{1}{N^{2}} \sum e^{i \vec{k} \overrightarrow{r_{j}}} \sum e^{-i \vec{k} \overrightarrow{r_{j}}}
$$

The position of the j-th electron $\overrightarrow{r_{j}}$ can be written as a sum of transverse and longitudinal parts $\overrightarrow{r_{j}}=r_{j t r}+z_{j}$. For longitudinal waves the transverse part of the $\mathrm{F}$ is equal to $N_{r}^{2}$. This is right because of transverse coherency of the radiation [3]. Note, that generally this expression must be averaged over all possible ensembles, or over $\mathrm{N}$-particle distribution function. But as seen from above formulae for coherency formfactor this one depend on two particle coordinates, so we can use for averaging the two particle distribution function. After such calculations formfactor in our notation is equal to

$$
F=F_{0}+F_{1},
$$

where $F_{0}$ is the usual formfactor [3] and $F_{1}$ is the additional term due of averaging over correlation function $\mathrm{g}$. This term describe the effect of correlations on the bunch coherency. For known correlation function formfactor of correlations can be calculated by averaging of the general formulae by correlation function. 


\subsection{Coherent Radiation of the Strong Correlated Uniform Bunch}

When the condition of crystallization or full ordering of the bunch is not satisfies, but the interparticle interaction is strong enough, the bunch becomes ordered with correlation function (1). In more simple notation we can write for factor $b$

$$
b=\frac{\lambda_{c}}{a \delta \sqrt{\Gamma(\gamma)^{3}}}
$$

where $\lambda_{c}$-Compton wavelength, $\delta=\frac{\Delta E}{E}$-mean energy deviation. It is not difficult to see, that for physical interesting range of parameters $b$ is less than $\lambda_{c} / a$, and much less than unity. Consequently, we can the neglect exponential dependence in this formula and use (2) for two particle distribution function. In this approach the longitudinal coherency formfactor is equal

$$
F=\frac{N_{r}^{2}}{N^{2}} \frac{\sin ^{2} N x}{x^{2}}
$$

\subsection{Coherent Radiation of the Ordered Bunch}

Consider now radiation of the ordered bunch. For $N_{z}$ planes with $N_{r}$ electrons on each plane the coherency factor is may be written in the following form

$$
F=\frac{N_{r}^{2}}{N} \sum e^{i k j \tilde{a}} \sum e^{-i k j \tilde{a}}
$$

where $\sum$-means sum over planes in longitudinal direction. It is not difficult to obtain that

$$
F=\frac{N_{r}^{2}}{N} \frac{\sin ^{2} N_{z} x}{\sin ^{2} x}
$$

and when the value $x=k a$ is approach to $n \pi$ (resonance condition) the coherency factor or laser gain becomes about $N$. This consideration is true for bunch with step function for longitudinal density distribution (for homogeneous or uniform bunch)

\subsection{The Influence of fluctuations}

Now consider the influence of fluctuations or deviations of the particle positions from hexagonal model above on the coherence short wave length radiation of the bunch. Let us assume that particle is on the mean distance, but not in the $\mathrm{z}$-direction. In this case the particle position is fluctuated on the value $a \theta^{2} / 2$, and if $x \theta^{2} / 4 \ll 1$ we may neglect this fluctuation. Notice, that in important practical cases this condition is satisfied. It is not satisfied only for very short radiation when $x \gg 1$. The second source of fluctuations from our model is possible, when the particle is replaced on the $\mathrm{z}$-direction but not in the distance 
a. In this case $\mathrm{F}$ multiplied by the factor $e^{-k^{2} b^{2}}$, where $\mathrm{b}$ is the dispersion of the fluctuation. It is clear that if $b / \lambda \ll 1$, this fluctuations not disturb the radiation coherency.Executed numerical calculations show that fluctuations about ten times smaller than inter particle distance $a$.

\section{Conclusions}

So, in this paper the influence of bunch particle position correlations on the bunch undulator radiation are investigated. Additional term in the radiation coherency formfactor was found. The correlations in the relativistic charge bunches are considered and are found the crystallization or ordering conditions of dense relativistic bunches or beams, which may be satisfied much easier than those for OCP. This very exotic and interesting state of matter can be obtained at existing linac beams. We show that ordered bunches can radiate coherently ,i.e. much powerful than spontaneous one. In dependence of beam energy and density this radiation may have wavelength at XUV range also.

\section{References}

[1] Pellegrini,C. et. al., Nucl.Inst. and Methods, 341(1994)326

[2] J. Rossbach,Nucl.Instr.and Methods,A 393(1997)86

[3] Korkhmazian,N.A. et.al., Zh.Tekh.Fiz. 47(1977)1583, Gevorgian,L.A., Zhevago,N.K., Sov.Phys.Dokl., 27(1982)946, in Russian

[4] Y.Pinhasi,A.Gover,Nucl.Instr. and Methods, A393(1997)343

[5] A.Gover et. al.,Phys.Rev.Lett.,72(1994)1192

[6] R.Bonifacio,B.W.J.McNeil,P.Pierini, Phys.Rev.A40(1989)4467

[7] R.Bonifacio,C.Maroli,N.Piovella, Optics Comm., 68(1988)369

[8] J.M.Ziman,'Models of disorder', Cambridge University Press,1979

[9] E.N.Dementiev,N.S.Dikansky,A.S.Medvedko, V.V.Parkhomchuk and D.V.Pestrikov, Zh.Tekh.Fiz. 50(1980)1717 [Sov.Phys.Tech.Phys. 25(1980)1001].

[10] A.V.Aleksandrov et al., Europhys.lett. 18(1992)151; M.Steck et al.,Electron Cooling at ESR, Workshop on Crystalline Beams and Related Issues, Erice, Sicily, 11-21 nov.1995,eds. D.M. Maletic and A.V.Ruggiero(World Scientific). 
[11] J.P.Schiffer and P.Kienle, Z.Phys. A321(1985)181; A.Rahman and J.P.Schiffer, Phys.Rev.Lett. 57(1986)1133; J.P.Schiffer, Phys.Rev.Lett. $61(1988) 1843$

[12] A.G.Ruggiero,Proceedings of the PAC93,p.3530

[13] W.L.Slattery,G.D.Dooley, and H.E.deWitt, Phys.rev. A21(1980)2087

[14] S.Ichimaru,H.Iyetomi,and S.Tanaka, Phys.Rep. 149(1987)93

[15] X.-P.Li,A.M.Sessler,and J.Wei, in Proceedings of the EPAC'04, p.1379. J.Wei,H.Okamoto,and A.M.Sessler, Phys.Rev.Lett., 80(1998)2606.

[16] H.J.Schulz,Phys.Rev.Lett.,71(1992)1864, E-print, cond-mat/9301007, 1993.

[17] Q.P.Li and S.D.Sarma, Phys.Rev, B43(1991)11768.

[18] F.Zimmermann and T.O.Raubenheimer, SLAC-PUB-7139, June 1996

[19] Ch. Settakorn, M. Hernandez, K. Woods, and H. Wiedemann, SLAC-PUB7813,1998 